\title{
Simulación matemática para la optimización del patrón de flujo entregado por una buza para el molde de colada continua de planchón delgado
}

\author{
Hugo Arcos Gutiérrez ${ }^{1}$, Gerardo Barrera Cardiel ${ }^{1}$, \\ Ramiro Escudero García ${ }^{1}$
}

\author{
${ }^{1}$ Instituto de Investigación en Metalurgia y Materiales, UMSNH, AP 888, Morelia, Mich. C.P. 58000, Morelia, Michoa- \\ cán, México. \\ e-mail: arcos.hugo@gmail.com \\ e-mail: gbarrera@umich.mx; ramgarci@umich.mx
}

\section{RESUMEN}

Entender el comportamiento de las oscilaciones de los chorros al interior del molde de planchón delgado tipo embudo es esencial para asegurar una entrega de acero líquido constante, mejorar el control de los patrones de flujo y consecuentemente incrementar la productividad de la planta y la calidad del producto final. Para lograr esto se llevó a cabo un estudio del efecto del diseño interno de la buza sobre la fluido dinámica del molde, intentando determinar el origen de las oscilaciones de los chorros. Se hizo uso de la simulación matemática para estudiar éstos fenómenos.

En la modelación matemática, se recurrió a las ecuaciones fundamentales, el modelo de turbulencia RSM y el modelo Multifásico VOF. Las ecuaciones gobernantes son discretizadas y resueltas mediante el método iterativo-segregado implícito implementado en FLUENT®.

Los resultados, de la simulación matemática, muestran que aun para diseños de buza con un comportamiento operacional estable, las oscilaciones de los chorros permanecen presentes y se hacen más intensos para altas velocidades de colada e inmersiones de buza profundas. El análisis de cada una de las buzas simuladas muestra que la geometría interna origina perturbaciones en el flujo en las zonas donde las áreas transversales internas cambian, generando presiones dinámicas altas y bajas promoviendo una tendencia para que el acero líquido salga preferentemente por uno de los puertos. Se encontró un delicado balance de fuerzas, el cual, se da sobre sobre la punta de la bifurcación interna de la buza. Este balance está relacionado con las velocidades fluctuantes y la presión ferrostática. Si este balance es roto las oscilaciones son más intensas, originando variaciones permanentes de la velocidad de flujo másico de un puerto a otro. Además, se encontró que existe la formación continua de un camino de vórtices el cual se genera a partir de la separación de la capa límite en el divisor de los puertos, fenómeno que intensifica la oscilación periódica de los chorros.

Palabras clave: Diseño de buza; simulación matemática; molde de planchón delgado tipo embudo; oscilación de los chorros; turbulencia.

\section{ABSTRACT}

Understanding the behavior of the oscillations of the jets into the mold of thin slab funnel is essential to ensure a constant supply of liquid steel, improve control of the flow patterns and consequently increase plant productivity and the final product quality. To achieve this, we conducted a study of the effect of the internal design of the nozzle on the fluid dynamics of the mold, trying to determine the origin of the oscillations of the jets. Use of mathematical and physical simulation was done to study these phenomena.

For the mathematical modeling it resorts to the fundamental equations, the RSM turbulence model and VOF multiphase model. The governing equations are discretized and solved by iteration-segregated implicit method implemented in FLUENT®.

The results of the mathematical simulation are showing that even for a nozzle designs with a stable operational performance, the oscillations of the jets remain present and become more intense for high casting speeds and deeper nozzle. The analysis of each of the simulated nozzles show that the internal geometry causes flow disturbances in areas where the internal cross-sectional areas change, generating high and low dynamic pres- 
sures and promoting a tendency for the liquid steel to exit preferably by one of the ports. A delicate balance of forces, in the order of micro-scales, was found and is given on the tip of the internal bifurcation of the nozzle. This balance is related to the fluctuating speeds and the ferrostatic pressure. If this balance is broken the oscillations are more severe, causing permanent changes in the mass flow rate from one port to another. In addition, it was found that there is continuous formation of a vortex path which is generated from the separation of the boundary layer on the splitter ports, a phenomenon that intensifies the periodic oscillation of the jets

Keywords: Nozzle design; mathematical simulation, funnel thin slab caster; jet oscillations; turbulence.

\section{INTRODUCCIÓN}

Con la creciente demanda de acero que existe hoy en día en el mundo y las rigurosas normas de calidad impuestas, exigidas por el mercado, a éste material, se ha vuelto un reto para las empresas siderúrgicas incrementar la producción existente sin sacrificar la calidad del acero. Por lo que, la colada continua de planchón delgado en moldes tipo embudo, ha ido posicionándose y teniendo gran aceptación debido a: la disminución en el espesor del planchón, a su relativamente alta velocidad de colada y a que en este proceso se llega al producto final en menos pasos comparado con la colada en moldes de planchón convencional. Sin embargo, éste proceso debe ser mejorado, ya que su alta velocidad de colada trae como consecuencia problemas operacionales, por lo que el estudio del flujo de fluidos juega un papel clave ya que por una parte la geometría compleja del molde y la buza de descarga involucran problemas asociados con alteraciones en el patrón de flujo generado al interior del molde ocasionando la oscilación de los chorros de descarga provenientes de los puertos de la buza, lo cual provoca la re-fusión de la capa de acero recién solidificada del planchón, así como fluctuación de la interfase metal-escoria [1-7]. Lo anterior, origina la presencia de otros fenómenos tales como vórticidades debido al flujo altamente turbulento existente al interior del molde [5-7, 13]. Estos fenómenos tienen un efecto negativo ocasionando que la capa de polvos lubricantes y transferencia de calor no sean homogéneos, exista atrapamiento de escoria hacia el seno de metal líquido y agrietamientos en la superficie del planchón producido [9-12].

Actualmente muchas de las investigaciones en el área parten de una buza que está siendo muy utilizada en la industria, llevando a cabo la caracterización de los patrones de flujo de la misma bajo ciertas condiciones operacionales tales como: velocidad de colada, profundidad de inmersión de buza y el ancho del planchón producido. En el presente estudio, se hace un análisis de dicha buza, la cual ha dado excelentes resultados a velocidades de colada bajas, $4 \mathrm{~m} / \mathrm{min}$. Se llevó el estudio hasta velocidades de colada extremas, $8 \mathrm{~m} / \mathrm{min}$, y haciendo el análisis para profundidades de inmersión 22 y $34 \mathrm{~cm}$. Se realizó una ligera modificación al diseño original de la buza, tanto en el interior como en los puertos, sin sacrificar la geometría externa de esta. La simulación matemática se llevó a cabo haciendo uso del código comercial de CFD Fluent.

\section{MODELO MATEMATICO}

Se desarrolló un modelo matemático para estudiar la fluidodinámica de dos buzas en el interior tanto de la buza como del molde de planchón delgado tipo embudo. A continuación, se hace una breve descripción de las diferentes ecuaciones y modelos utilizados.

\subsection{Ecuaciones fundamentales}

Las ecuaciones fundamentales que gobiernan este modelo son las ecuaciones de Navier-Stokes y de continuidad para fluidos incompresibles en coordenadas cartesianas, y se escriben como sigue ${ }^{[15-19]}$.

$(\nabla \cdot \mathrm{u})=0$

$\rho[\nabla \cdot u u]=-\nabla P+\mu_{\text {eff }} \nabla^{2} u+\rho g$

Modelo k- $\varepsilon$ estándar ${ }^{\text {[13-17] }}$

Este modelo es definido como sigue:

$\rho \frac{\partial \mathrm{k}}{\partial \mathrm{t}}+\rho \frac{\partial \mathrm{ku}_{\mathrm{i}}}{\partial \mathrm{x}_{\mathrm{i}}}=\frac{\partial}{\partial \mathrm{x}_{\mathrm{j}}}\left[\left(\mu+\frac{\mu_{\mathrm{t}}}{\sigma_{\mathrm{k}}}\right) \frac{\partial \mathrm{k}}{\partial \mathrm{x}_{\mathrm{j}}}\right]+\mathrm{G}_{\mathrm{k}}-\rho \varepsilon$ 
$\rho \frac{\partial \varepsilon}{\partial t}+\rho \frac{\partial u_{i}}{\partial x_{i}}=\frac{\partial}{\partial x_{j}}\left[\left(\mu+\frac{\mu_{t}}{\sigma_{\varepsilon}}\right) \frac{\partial \varepsilon}{\partial x_{j}}\right]+C_{1 \varepsilon} \frac{\varepsilon}{k} G_{k}-C_{2 \varepsilon} \rho \frac{\varepsilon^{2}}{k}$

Siendo $C_{1}, C_{2}, \sigma_{k}, \sigma_{\varepsilon}$ constantes cuyos valores son $1.44,1.92,1.0$ y 1.3 , respectivamente. Modelo de Turbulencia RSM ${ }^{[15-19]}$

$$
\begin{aligned}
& \frac{\partial}{\partial \mathrm{t}}\left(\rho\left\langle\mathrm{U}_{\mathrm{i}}^{\prime} \mathrm{U}_{\mathrm{j}}^{\prime}\right\rangle\right)+\left(\rho_{\mathrm{k}}\left\langle\mathrm{U}_{\mathrm{i}}^{\prime} \mathrm{U}_{\mathrm{j}}^{\prime}\right\rangle\right)=\frac{\partial}{\partial \mathrm{X}_{\mathrm{k}}}\left[\rho\left\langle\mathrm{U}_{\mathrm{i}}^{\prime} \mathrm{U}_{\mathrm{j}}^{\prime} \mathrm{U}_{\mathrm{k}}^{\prime}\right\rangle+\left\langle\mathrm{P}\left(\delta_{\mathrm{kj}} \mathrm{U}_{\mathrm{i}}^{\prime}+\delta_{\mathrm{jk}} \mathrm{U}_{\mathrm{j}}^{\prime}\right)\right\rangle\right]+ \\
& \frac{\partial}{\partial \mathrm{X}_{\mathrm{k}}}\left[\mu \frac{\partial}{\partial \mathrm{X}_{\mathrm{k}}}\left\langle\mathrm{U}_{\mathrm{i}}^{\prime} \mathrm{U}_{\mathrm{j}}^{\prime}\right\rangle\right]-\rho\left(\left\langle\mathrm{U}_{\mathrm{i}}^{\prime} \mathrm{U}_{\mathrm{j}}^{\prime}\right\rangle \frac{\partial \mathrm{U}_{\mathrm{j}}}{\partial \mathrm{X}_{\mathrm{k}}}+\left\langle\mathrm{U}_{\mathrm{i}}^{\prime} \mathrm{U}_{\mathrm{j}}^{\prime}\right\rangle \frac{\partial \mathrm{U}_{\mathrm{i}}}{\partial \mathrm{X}_{\mathrm{k}}}\right)-\rho \beta\left(\mathrm{g}_{\mathrm{i}}\left\langle\mathrm{U}_{\mathrm{j}}^{\prime} \theta\right\rangle+\mathrm{g}_{\mathrm{j}}\left\langle\mathrm{U}_{\mathrm{i}}^{\prime} \theta\right\rangle\right)+ \\
& \left\langle\mathrm{P}\left(\frac{\partial \mathrm{U}_{\mathrm{i}}}{\partial \mathrm{X}_{\mathrm{j}}}+\frac{\partial \mathrm{U}_{\mathrm{j}}}{\partial \mathrm{X}_{\mathrm{i}}}\right)\right\rangle-2 \mu\left\langle\frac{\partial \mathrm{U}_{\mathrm{i}}}{\partial \mathrm{X}_{\mathrm{k}}}+\frac{\partial \mathrm{U}_{\mathrm{j}}}{\partial \mathrm{X}_{\mathrm{k}}}\right\rangle-2 \rho \Omega_{\mathrm{k}}\left(\left\langle\mathrm{U}_{\mathrm{j}}^{\prime} \mathrm{U}_{\mathrm{m}}^{\prime}\right\rangle \varepsilon_{\mathrm{ikm}}+\left\langle\mathrm{U}_{\mathrm{i}}^{\prime} \mathrm{U}_{\mathrm{m}}^{\prime}\right\rangle \varepsilon_{\mathrm{jkm}}\right)
\end{aligned}
$$

Y el modelo de Volumen de Fluido (VOF), fue empleado para el cálculo de la interface en el sistema agua/aire. El modelo emplea una técnica de rastreo diseñada para dos o más fases de fluidos inmiscibles, donde la posición de la interfase entre los fluidos es de interés. Este modelo ha sido ampliamente usado en trabajos previos y ha sido descrito completamente en la literatura abierta [13-14].

\subsection{Consideraciones del modelo}

Con la finalidad de realizar una validación de los resultados del modelo numérico con los posteriores resultados del modelo físico, se simuló agua como fluido de trabajo en la simulación numérica. Todas las simulaciones se llevaron a cabo en estado transitorio e isotérmico. Se consideraron condiciones de no deslizamiento en todas las paredes sólidas del modelo. La entrada y salida fueron consideradas como entrada de velocidad, esto para mantener la velocidad de colada constante en la salida del molde. En la parte superior del molde se consideró al modelo abierto a la atmósfera, por lo cual se tiene una entrada de presión de 101325 Pa. La gravedad solo actuará en la dirección de colada. Las propiedades físicas del fluido y los parámetros de la simulación son mostradas en la Tabla 1, dónde SEN significa Submerged Entry Nozzle por sus siglas en inglés.

Tabla 1: Propiedades físicas.

\begin{tabular}{ccc}
\hline Región del Modelo & Propiedad & Valor \\
\hline Aire & Densidad $\left(\mathrm{kg} / \mathrm{m}^{3}\right)$ & 1.225 \\
& Viscosidad $(\mathrm{Pa} \mathrm{s})$ & $1.7894 \times 10^{-5}$ \\
Agua & Densidad $\left(\mathrm{kg} / \mathrm{m}^{3}\right)$ & 984 \\
& Viscosidad $(\mathrm{Pa} \mathrm{s})$ & 0.001003 \\
Aire-Agua & Tensión Superficial $(\mathrm{N} / \mathrm{m})$ & 0.075 \\
Altura de la & Superficial $(\mathrm{cm})$ & 22 \\
SEN en el molde & Profunda $(\mathrm{cm})$ & 34 \\
\hline
\end{tabular}

Para lograr una mayor exactitud en los resultados, primeramente, se resolvió durante un minuto el modelo numérico empleando el modelo de turbulencia k- $\varepsilon$, para posteriormente cambiar al modelo de Esfuerzos de Reynolds (RSM) y continuar con la simulación hasta alcanzar seis minutos, con lo cual se buscó alcanzar una etapa de estado estable. Las ecuaciones del modelo se discretizaron a través del software computacional FLUENT empleando el método segregado con una formulación implícita. Además, se estableció un primer orden en la discretización de las ecuaciones para el modelo de turbulencia y las ecuaciones Navier-Stokes; además de resolver las ecuaciones de transporte para el movimiento, es requerido que las presiones en las caras de las celdas sean conocidas. Al igual que con las demás cantidades transportadas, lo cual, es alcanzado mediante interpolación de los valores en las celdas vecinas, por lo cual, se utilizó el algoritmo Body Force Weighted para calcular la presión y el método SIMPLEC, este algoritmo es usado como una suposición para que la presión y la velocidad sea resuelta para las velocidades correspondientes a las ecuaciones de movimiento. Puesto que la suposición de inicio de la presión no será la correcta, por lo que, las velocidades obtenidas no satisfacen la continuidad. Por lo tanto, los factores de corrección para la presión y la velocidad son introducidos y las ecuaciones de transporte para esos factores son propuestas y resueltas para dar velocidades y presiones corregidas. El criterio o tolerancia de convergencia fue igual o menor a $1 \times 10^{-4}$

En todos los casos se consideraron constantes el ancho y espesor del molde, pero se manejaron dos inmersiones de la buza (la mínima y máxima permisibles) y se simularon cinco velocidades diferentes, desde los valores mínimos hasta los máximos deseados para operación en planta. Se simularon diez casos utilizando el diseño original de la buza. Con estos resultados se modificó el diseño de la buza y se simularon diez casos 
más, en donde las alturas de la SEN en el molde fueron de 22 y $34 \mathrm{~cm}$ por debajo de la superficie del baño, estos se presentan en la Tabla 2.

Tabla 2: Casos de estudio.

\begin{tabular}{ccc}
\hline Caso & $\begin{array}{c}\text { Diseño } \\
\text { SEN }\end{array}$ & $\begin{array}{c}\text { Velocidad de colada } \\
(\mathbf{m} / \mathbf{m i n})\end{array}$ \\
\hline $\mathbf{1}$ & Original & 4 \\
$\mathbf{2}$ & Original & 5 \\
$\mathbf{3}$ & Original & 6 \\
$\mathbf{4}$ & Original & 7 \\
$\mathbf{5}$ & Original & 8 \\
$\mathbf{6}$ & Modificada & 4 \\
$\mathbf{7}$ & Modificada & 5 \\
$\mathbf{8}$ & Modificada & 6 \\
$\mathbf{9}$ & Modificada & 7 \\
$\mathbf{1 0}$ & Modificada & 8 \\
\hline
\end{tabular}

La Figura 1 muestra esquemáticamente los parámetros geométricos más importantes del molde tipo embudo y las dos buzas que fueron utilizadas en el presente trabajo de investigación, en dicha figura se presentan las vistas frontales de las mismas. La modificación se llevó a cabo en la geometría interna de la buza original, la cual consistió en eliminar el escalón en la parte superior de ambos puertos y en incrementar la altura de la división de estos. El dominio computacional fue dividido en 1,600,000 celdas, haciendo uso de una malla no estructurada.

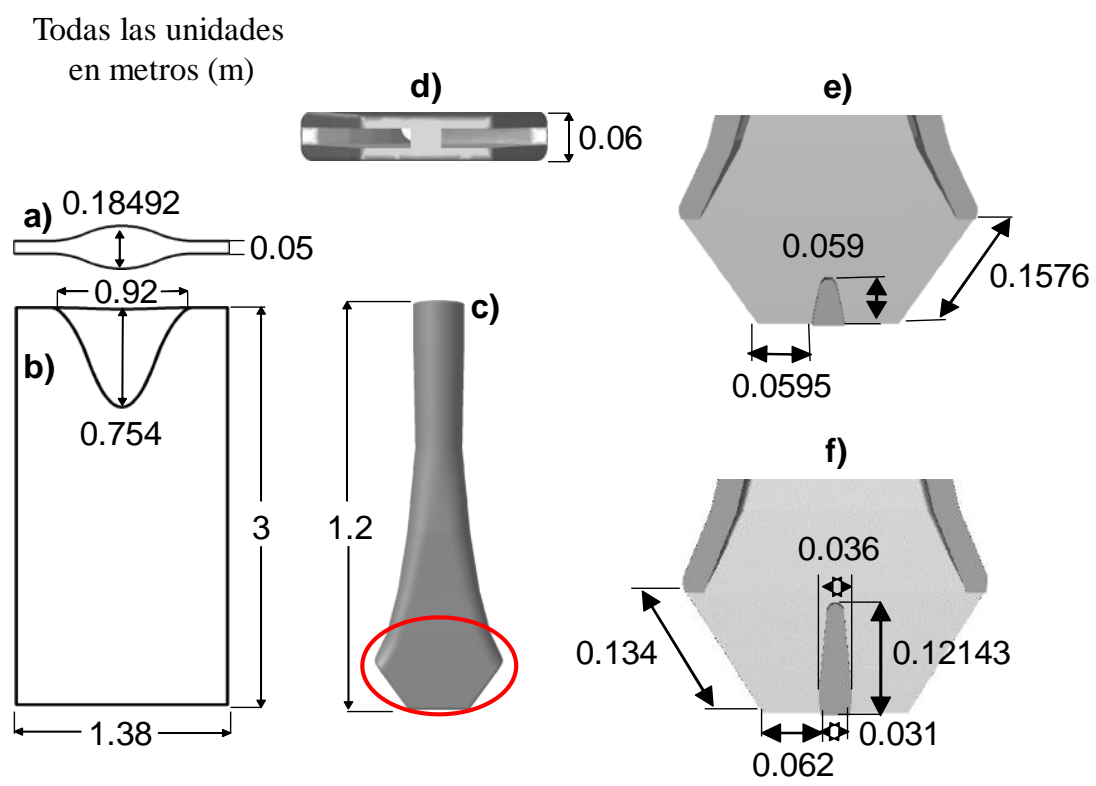

Figura 1: Esquema de la geometría para: a)-b) molde de planchón delgado tipo embudo, c)-d) la SEN, e) interna SENoriginal, and f) interna SEN-modificada.

\section{Análisis y Discusión de los Resultados}

A continuación, se lleva a cabo el análisis y discusión de los resultados obtenidos comparando una buza utilizada actualmente en la industria y una mejorada a partir de esta (modificada). El análisis se hace a partir de la medición de la producción de energía cinética, así como de la relación de disipación turbulenta, de intensidad turbulenta y magnitud de la vórticidad. Para con esto tener un entendimiento más preciso del efecto de cada una de estas variables sobre la estabilidad de los chorros provenientes de los puertos de la buza y el efecto de estos sobre los patrones de flujo generados al interior del molde y la buza. La medición de cada una de las variables mencionadas anteriormente se llevó a cabo trazando una línea al centro del sistema buza-molde a inmersiones de buza de $22 \mathrm{~cm}$ y $34 \mathrm{~cm}$ y velocidades de colada de 4,6 y $8 \mathrm{~m} / \mathrm{min}$. 


\subsection{Efecto de la geometría interna de la buza sobre los fenómenos de flujo de fluidos presentados al interior del molde}

En trabajos previos $[11,13]$ se ha sugerido que la formación de Distorsiones Dinámicas (DD) en moldes para planchón delgado es una consecuencia del desequilibrio en el balance de la energía cinética turbulenta y su producción, principalmente en la zona ubicada en la parte inferior de la buza. En estos trabajos se realizó un análisis de cómo se lleva a cabo la DD y se ha dejado planteado la hipótesis antes mencionada, la cual permanece aún sin comprobación. Éstos plantean que los orígenes de las DD podrían iniciarse desde el interior de la buza, ya que se supone que ésta entrega el flujo de acero en cada puerto de una manera equilibrada. Se plantea la hipótesis de que fluctuaciones en la alimentación en los puertos favorecerán la formación de las DD. Sin embargo, en el presente trabajo de investigación se plantea una nueva hipótesis, la cual, refuta la primera, dado que se propone que el origen de las DD tiene su origen en el interior de la buza debido a fluctuaciones en la presión y velocidad en la zona por encima del divisor de los puertos, y que suprimiendo la zona del fluido por debajo de la punta de la buza que es en donde se origina un camino de vórtices ocasionado por la separación de la capa limite en el divisor de los puertos, fenómeno que aunado a la vórticidad proveniente del fondo del molde provoca la falta de simetría existente en los chorros y la posterior oscilación de estos sobre todo a velocidades de colada altas y una de inmersión de buza profunda.

Para llevar a cabo la comprobación de esta hipótesis, se seleccionó una buza cuya operación fuera estable y sus resultados fueron comparados con los de una buza modificada a partir de los resultados obtenidos con la primera, se experimentó con dos diferentes profundidades de inmersión y con diferentes velocidades de colada con las que la formación de DD son muy probables. La Fig. 2 muestra los perfiles de velocidad calculados al centro del molde en un plano longitudinal, para las 5 velocidades de colada seleccionadas a $22 \mathrm{~cm}$ de inmersión. Los patrones de flujo son característicos para este tipo de molde, mostrando la formación de una gran recirculación a cada lado de la buza y zonas de baja velocidad en las posiciones de: las esquinas en la parte superior del molde, en las cercanías a la buza y en la zona por debajo de la buza entre los dos chorros. La Fig. 2 a) muestra que la estabilidad de los chorros a una velocidad de colada de $4 \mathrm{~m} / \mathrm{min}$ es muy buena, pero conforme se incrementa la velocidad de colada los chorros muestran una variación en su patrón de flujo.

Esta variación se observa en que el chorro izquierdo (marcado con el número (1)) presenta una penetración más intensa que el derecho. Esta variación inicia a una velocidad de $5 \mathrm{~m} / \mathrm{min}$ y se mantiene hasta la velocidad máxima de $8 \mathrm{~m} / \mathrm{min}$. Cuando la buza es colocada en la inmersión de $34 \mathrm{~cm}$, Fig. 3, se observa el típico patrón de flujos de planchón delgado a una velocidad de 4 y $5 \mathrm{~m} / \mathrm{min}$; sin embargo, conforme se incrementa la velocidad de colada se observa que los chorros comienzan a oscilar, pero en este caso es el chorro izquierdo el que se presenta mayor desviación (Figs. 3 c) y 4 d), punto (1) y posteriormente esto se revertirá hacia el derecho (Fig. 3 e) punto(2)). Además, se puede apreciar que el espacio entre ambos chorros a ambas profundidades de inmersión de buza y a las distintas velocidades de colada, se da un intercambio de energía entre la vórticidad generada debido a la separación de la capa limite en el divisor de los puertos y la vórticidad proveniente de la recirculación proveniente del fondo del molde. En las figuras 2, 3, 4 y7, la notación o tamaño del vector significa la magnitud de la velocidad, vectores más pequeños significa que la velocidad es menor a ésta referencia y más grandes velocidades más altas. 

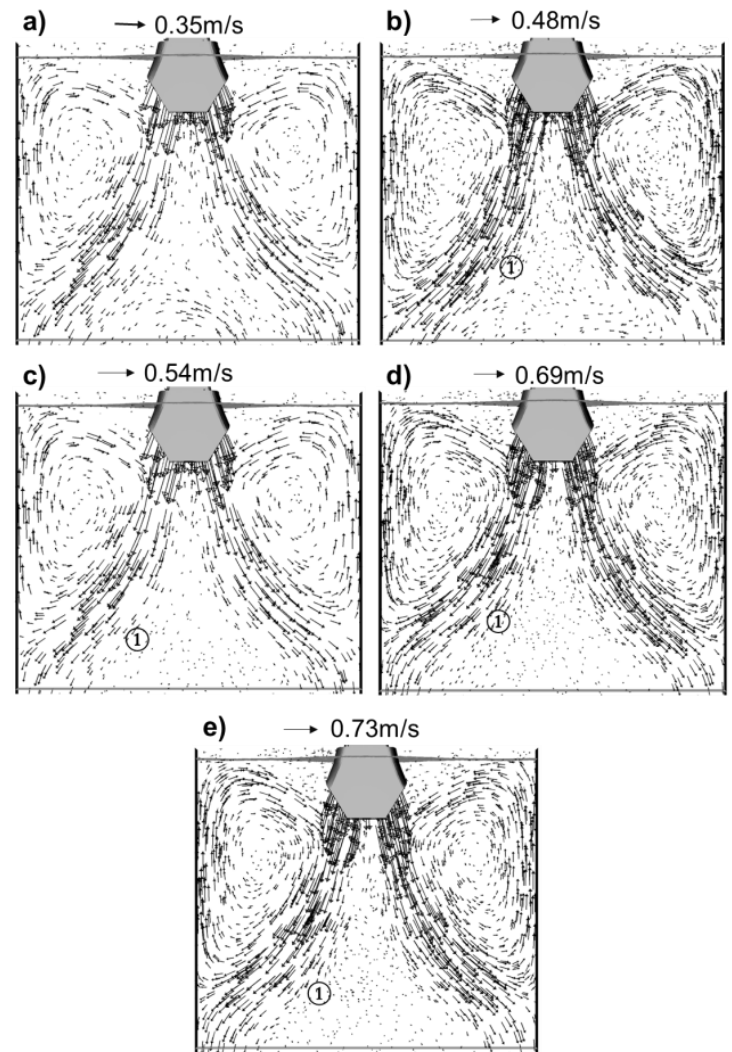

Figura 2: Vectores de velocidad en un plano simétrico central del molde a inmersión de buza de $22 \mathrm{~cm}$ y velocidades de colada de: a) $4 \mathrm{~m} / \mathrm{min}$, b) $5 \mathrm{~m} / \mathrm{min}$, c) $6 \mathrm{~m} / \mathrm{min}$, d) $7 \mathrm{~m} / \mathrm{min}$ y e) $8 \mathrm{~m} / \mathrm{min}$.
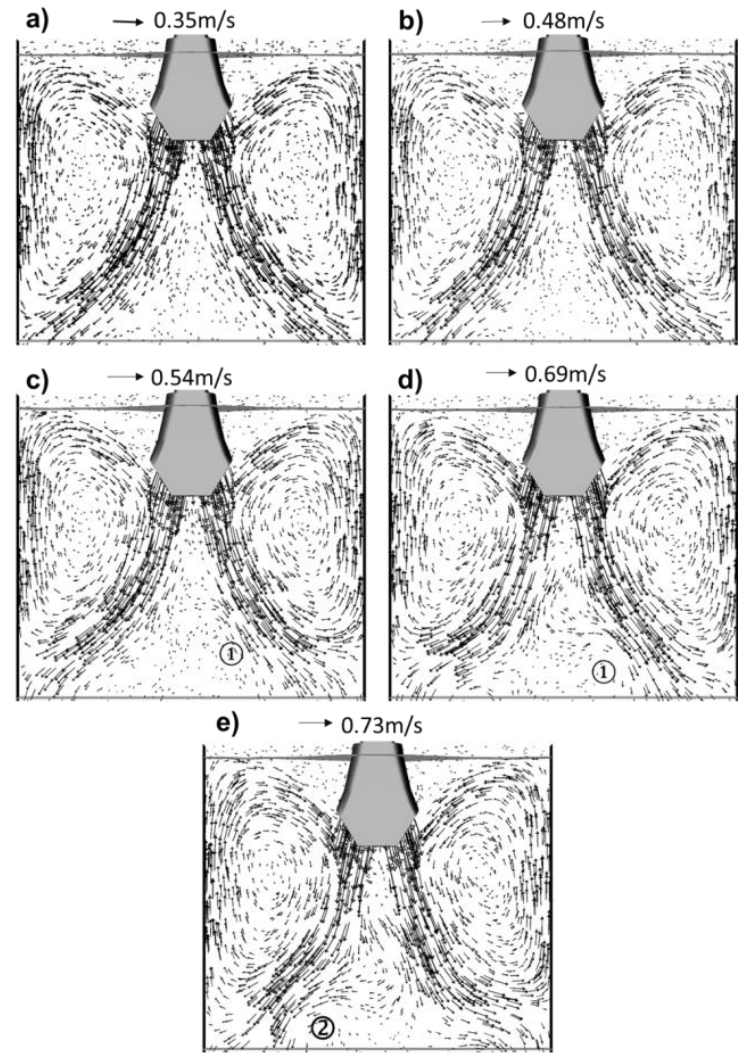

Figura 3: Vectores de velocidad en un plano simétrico central del molde a inmersión de buza de $34 \mathrm{~cm}$ y velocidades de colada de: a) $4 \mathrm{~m} / \mathrm{min}$, b) $5 \mathrm{~m} / \mathrm{min}$, c) $6 \mathrm{~m} / \mathrm{min}$, d) $7 \mathrm{~m} / \mathrm{min}$ y e) $8 \mathrm{~m} / \mathrm{min}$. 
A una mayor inmersión, las oscilaciones de los chorros son más evidentes. La variación en la inmersión de los chorros es más fácilmente observada y cuantificada considerando el punto de impacto de los mismos sobre la cara angosta del molde. Para observar esta variación, se trazó una línea imaginaria a lo largo del molde en las paredes derecha e izquierda de las caras angostas, donde se determinaron las velocidades del acero para 22 y $34 \mathrm{~cm}$ de inmersión de buza. Las Figs. 4 y 5 muestran como el perfil de velocidades varía, a lo largo del molde, dependiendo de la velocidad de colada y la profundidad de la buza. A mayor velocidad de colada y mayor profundidad de la buza los chorros oscilan más, lo cual, se ve reflejado en la zona de impacto de estos sobre la pared delgada del molde. Además, los flujos de acero son menores en las zonas cercanas a las paredes del molde y desplazándose a la parte media del molde, favoreciendo el crecimiento de la capa solidificada y por ende un mejor control de la colada. La longitud metalúrgica del molde se refiere al tamaño del molde en el sentido vertical del mismo, el molde mide $1.2 \mathrm{~m}$, es decir, la longitud metalúrgica del molde es $1.2 \mathrm{~m}$.

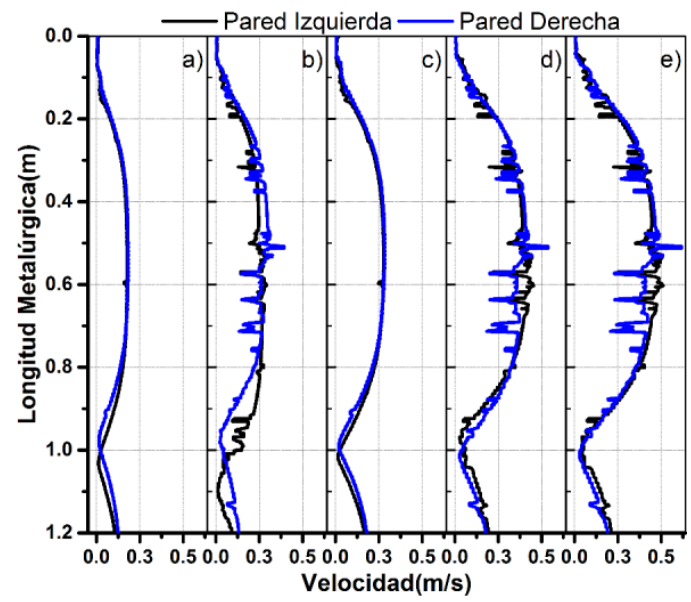

Figura 4: Perfil de velocidad en una línea imaginaria trazada sobre las paredes derecha e izquierda de la sección angosta del molde a una inmersión de buza de $22 \mathrm{~cm}$. a) $4 \mathrm{~m} / \mathrm{min}$, b) $5 \mathrm{~m} / \mathrm{min}$, c) $6 \mathrm{~m} / \mathrm{min}$, d) $7 \mathrm{~m} / \mathrm{min}$ y e) $8 \mathrm{~m} / \mathrm{min}$.

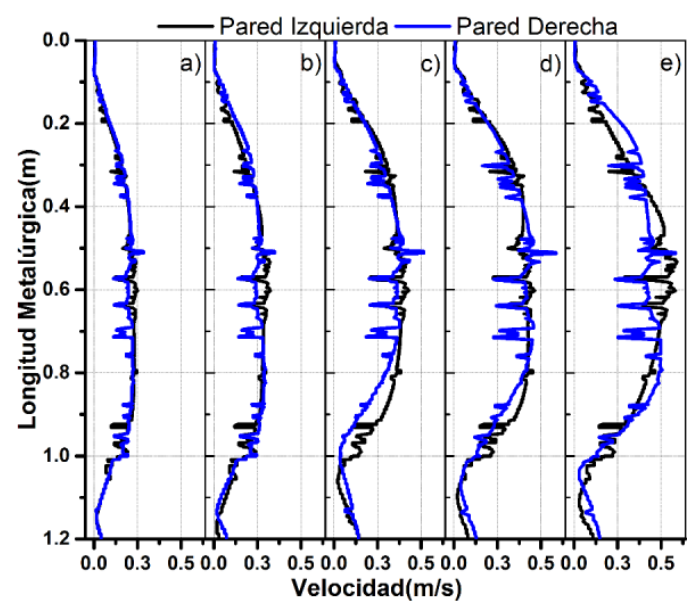

Figura 5: Perfil de velocidad en una línea trazada sobre la cara estrecha del molde a inmersión de buza de $34 \mathrm{~cm}$. a) $4 \mathrm{~m} / \mathrm{min}$, b) $5 \mathrm{~m} / \mathrm{min}$, c) $6 \mathrm{~m} / \mathrm{min}$, d) $7 \mathrm{~m} / \mathrm{min}$ y e) $8 \mathrm{~m} / \mathrm{min}$.

\subsection{Efectos de la modificación interna de la SEN sobre los patrones de flujo}

Ahora, se plantea una modificación en la geometría interna de la buza, que sea capaz de modificar el patrón de flujo al interior del molde, en la cual, el divisor de los puertos se hizo más alto, respecto al de la buza original, un $106 \%$ más alto, Fig. 1. Además, se le dio a éste una forma trapezoidal invertida. En lo que respecta a la parte inferior de los puertos, es decir, la sección de salida, también se incrementó en 2.5 mm compensando con esto la incorporación de la sección trapezoidal del divisor de los puertos. Con esto se pretende eliminar la zona, por debajo de la punta de la buza, donde se originan las distorsiones dinámicas, obligando a que los chorros salientes de la buza compartan su energía creando un balance entre ambos, eliminando además el efecto de la presión ferrostática y las oscilaciones. 
Las Figs. 6 y 7 muestran los patrones de flujo para 22 y $34 \mathrm{~cm}$ de inmersión de buza. En la zona marcada con el número (1) se logra invertir la dirección de los vectores ya que estos van en el sentido del flujo de los chorros, evitando con esto que el fluido recirculante proveniente del fondo del molde llegue a impactar la punta de la buza logrando con esto eliminar la formación del camino de vórtices provocado por la separación inestable de la capa límite con el divisor de los puertos, como sucede con la buza original. Este fenómeno se debe a que la geometría interna de ésta buza direcciona el flujo proveniente de los puertos hacia el centro del molde compartiendo así su energía hasta que la presión ferrostática hace que éstos se separen y se incorporen a las recirculaciones inferiores y superiores del molde. Además, se puede apreciar que al igual que el patrón de flujo está compuesto por dos recirculaciones en la zona superior del molde y dos en la parte inferior de este, al igual que con la buza original, solo que a diferencia de la primera esta presenta una buena simetría en el comportamiento de los chorros provenientes de la buza en las distintas velocidades y en ambas inmersiones de buza.

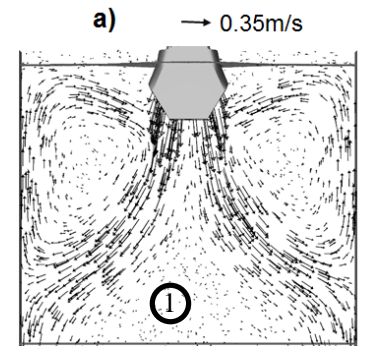

c) $\longrightarrow 0.54 \mathrm{~m} / \mathrm{s}$

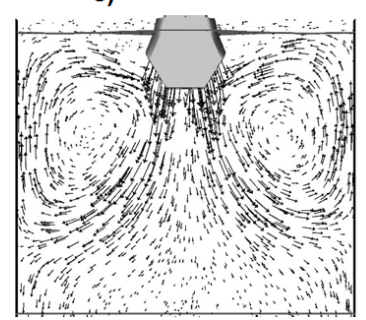

e) b) $\quad \rightarrow 0.48 \mathrm{~m} / \mathrm{s}$

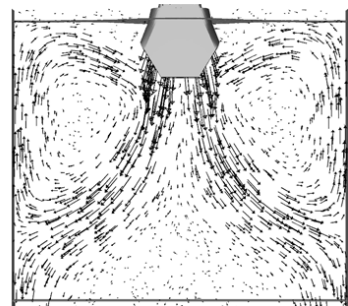

d) $\rightarrow 0.69 \mathrm{~m} / \mathrm{s}$

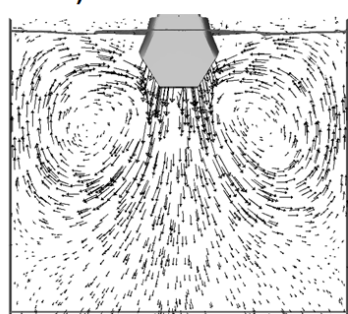

$\rightarrow 0.73 \mathrm{~m} / \mathrm{s}$

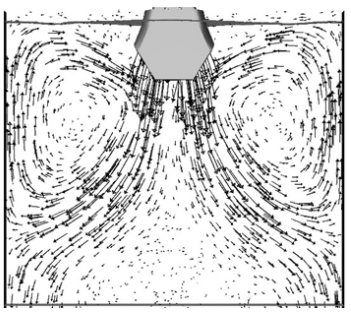

Figura 6: Vectores de velocidad en un plano simétrico central del molde, buza modificada. Inmersión de buza de $22 \mathrm{~cm}$ y velocidades de colada de: a) $4 \mathrm{~m} / \mathrm{min}$, b) $5 \mathrm{~m} / \mathrm{min}$, c) $6 \mathrm{~m} / \mathrm{min}$, d) $7 \mathrm{~m} / \mathrm{min}$ y e) $8 \mathrm{~m} / \mathrm{min}$. 


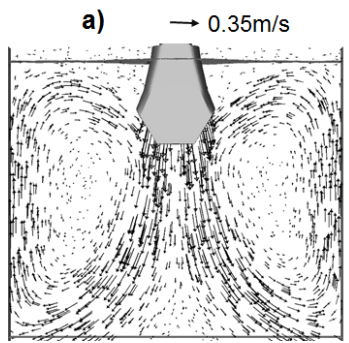

c) $\longrightarrow 0.54 \mathrm{~m} / \mathrm{s}$

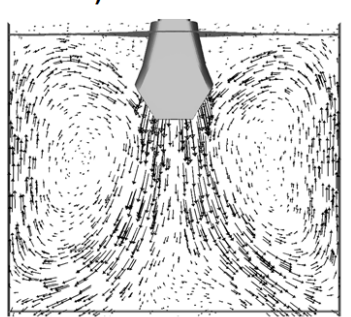

e) b) $\rightarrow 0.48 \mathrm{~m} / \mathrm{s}$

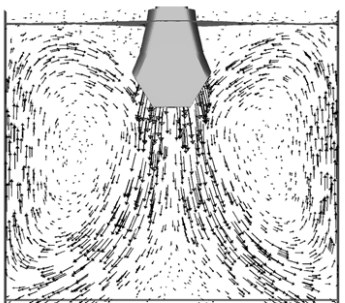

d) $\quad \rightarrow 0.69 \mathrm{~m} / \mathrm{s}$

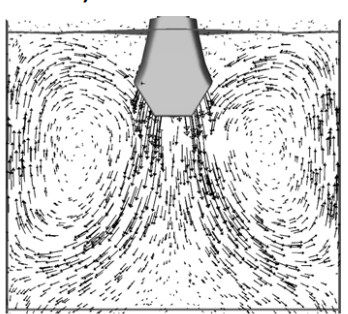

$\rightarrow 0.73 \mathrm{~m} / \mathrm{s}$

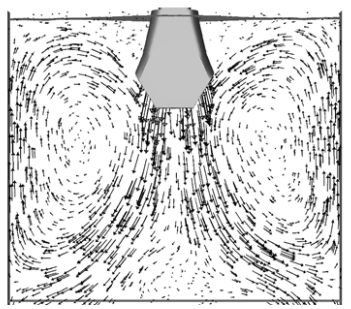

Figura 7: Vectores de velocidad en un plano simétrico central del molde, buza modificada. Inmersión de buza de 34cm y velocidades de colada de: a) $4 \mathrm{~m} / \mathrm{min}$, b) $5 \mathrm{~m} / \mathrm{min}$, c) $6 \mathrm{~m} / \mathrm{min}$, d) $7 \mathrm{~m} / \mathrm{min}$ y e) $8 \mathrm{~m} / \mathrm{min}$

En cuanto a la penetración de los chorros a $22 \mathrm{~cm}$ de inmersión de buza los chorros de la buza modificada penetran ligeramente más, esto para velocidades de colada de 4,5 y $6 \mathrm{~m} / \mathrm{min}$. Para velocidades de 7 y 8 $\mathrm{m} / \mathrm{min}$ por perfiles son muy similares, auqnue para $8 \mathrm{~m} / \mathrm{min}$ las velocidades sonmás altas en la sección media de la longitud metalúrgica del molde. Para $34 \mathrm{~cm}$ de inmersión de buza, Fig. 9, ambos chorros penetran a una profundidad similar. Existiendo diferencias marcadas sobre todo en el patrón de flujo entregado por cada una de éstas ya que la buza original oscila en prácticamente todas las velocidades de colada utilizadas, mientras que la geometría modificada es bastante estable. Se puede apreciar claramente que la separación de los chorros es un factor importante en la aparición de la DD (camino de vórtices) (1), ya que si esta zona no es suprimida existe un continuo intercambio de energía entre un chorro y otro, esto sin menospreciar la transferencia de energía de la vórticidad proveniente del fondo del molde. Lo cual, toma mayor fuerza mientras se incrementa la velocidad de colada y la profundidad de inmersión de buza.

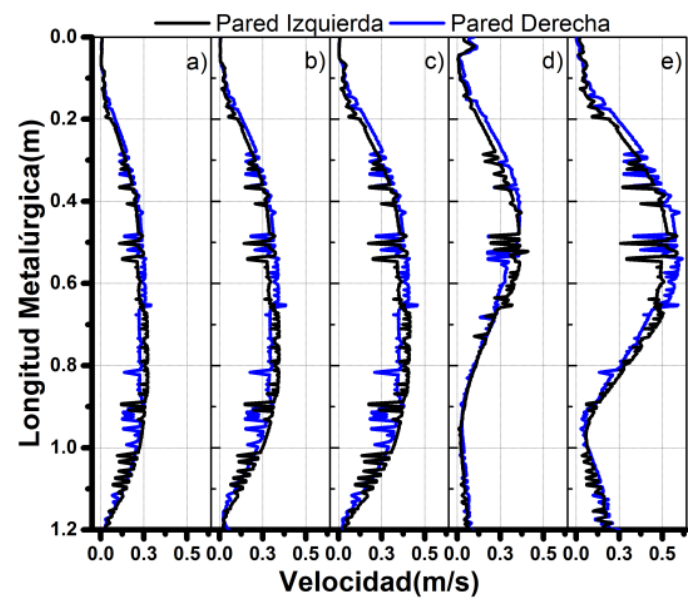

Figura 8: Perfil de velocidad en una línea trazada sobre la cara estrecha del molde a inmersión de buza de $22 \mathrm{~cm}$. a) $4 \mathrm{~m} / \mathrm{min}$, b) $5 \mathrm{~m} / \mathrm{min}$, c) $6 \mathrm{~m} / \mathrm{min}$, d) $7 \mathrm{~m} / \mathrm{min}$ y e) $8 \mathrm{~m} / \mathrm{min}$. 


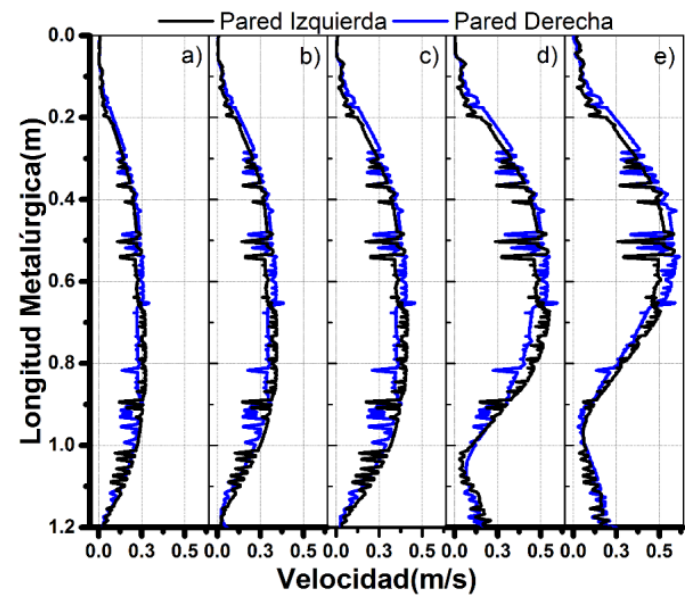

Figura 9: Perfil de velocidad en una línea trazada sobre la cara estrecha del molde a inmersión de buza de $34 \mathrm{~cm}$. a) $4 \mathrm{~m} / \mathrm{min}$, b) $5 \mathrm{~m} / \mathrm{min}$, c) $6 \mathrm{~m} / \mathrm{min}$, d) $7 \mathrm{~m} / \mathrm{min}$ y e) $8 \mathrm{~m} / \mathrm{min}$.

\subsection{Comparación del efecto de la modificación interna de la geometría original de la buza sobre el me- nisco}

Los resultados arrojan que a velocidades de colada altas y a una mayor profundidad de inmersión, en la buza original, las oscilaciones aumentan, coincidiendo con un incremento en las distorsiones dinámicas. Bajo las mismas condiciones la buza modificada se comportó mejor, manteniendo un patrón de flujo más estable. En la Fig. 10 para $22 \mathrm{~cm}$ de inmersión de buza y para las distintas velocidades de colada a) $4 \mathrm{~m} / \mathrm{min}$, b) $6 \mathrm{~m} / \mathrm{min}$ y c) $8 \mathrm{~m} / \mathrm{min}$ ), se puede apreciar claramente que la buza modificada al originar una mejor simetría y estabilidad de los chorros de descarga, además de que prácticamente no existen oscilaciones de los mismos, se alimenta mejor al menisco, comportamiento que no provoca inestabilidad en la interfase o fluctuaciones de los mismos.

Para la profundidad de inmersión de la buza de $34 \mathrm{~cm}$, Fig. 11, la buza modificada, debido a las oscilaciones menos intensas de los chorros y al espacio entre éstos, permite la generación del camino de vórtices que dan lugar a una mejor alimentación al menisco, comparada con lo que ocurre en la buza original.

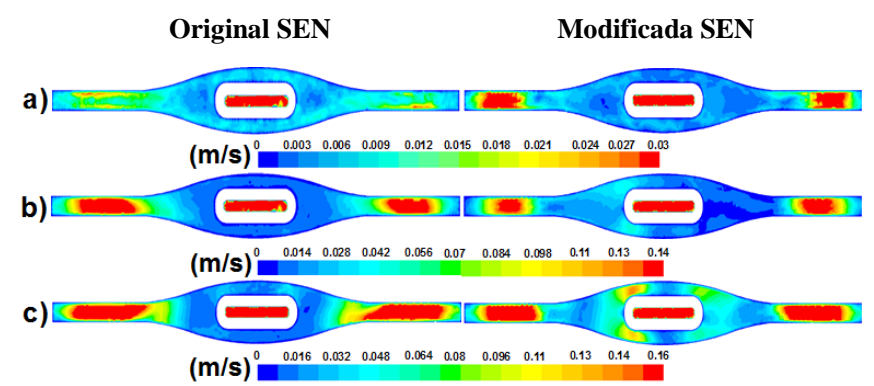

Figura 10: Contorno de velocidad a $22 \mathrm{~cm}$ de inmersión de buza. a) $4 \mathrm{~m} / \mathrm{min}$, b) $6 \mathrm{~m} / \mathrm{min}$, c) $6 \mathrm{~m} / \mathrm{min}$.

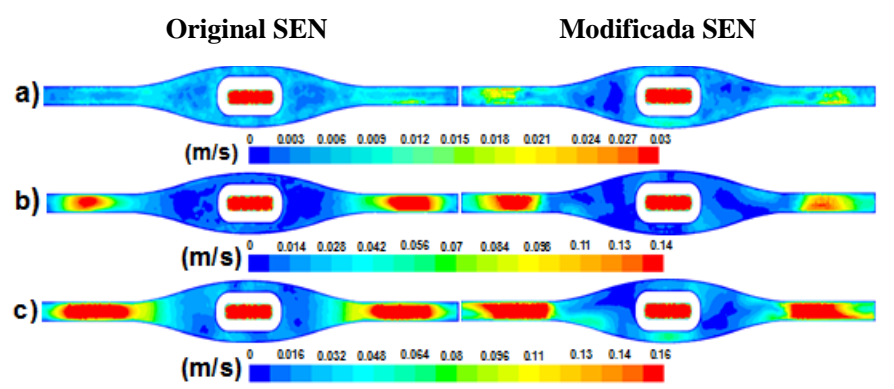

Figura 11: Contorno de velocidad a $34 \mathrm{~cm}$ de inmersión de buza. a) $4 \mathrm{~m} / \mathrm{min}$, b) $6 \mathrm{~m} / \mathrm{min}$, c) $6 \mathrm{~m} / \mathrm{min}$. 


\section{CONCLUSIONES}

Se llevó a cabo una simulación matemática para observar los efectos de la modificación de la geometría interna de la buza sumergida original SEN sobre la fluido dinámica y los patrones de flujo. De los resultados obtenidos se llegó a las siguientes conclusiones:

Existen buzas comerciales que se presentan oscilaciones de los chorros en los puertos de salida. esas oscilaciones se incrementan al aumentar tanto la velocidad de colada como la profundidad de inmersión de la buza. Solamente a muy altas velocidades de colada se pueden observar distorsiones dinámicas menores, por lo que se deduce que las oscilaciones de los chorros tienen su origen al interior de la SEN.

El diseño de la geometría interna de la buza provoca perturbaciones en el flujo, provocando que el acero líquido salga de manera preferencial por alguno de los puertos. Se encontró, además, que el tamaño de las zonas de presión es una función inversa de la velocidad de colada.

Se da un balance de fuerzas en la punta de la bifurcación interna de la buza. Este balance está directamente relacionado con el flujo fluctuante en dicha zona. Cuando éste balance es interrumpido, debido a la posición de la buza y/o por altas velocidades de colada, las oscilaciones son más evidentes e intensas cuando la buza se posiciona a mayor profundidad promoviendo variaciones permanentes en el flujo másico de un puerto al otro.

Sí se suprime la zona donde se forma un camino de vórtices (distorsión dinámica) que va desde la punta de la buza hacia el fondo del molde y se hacen converger los chorros justo al centro del molde, las oscilaciones de los chorros son mejor controlados, lo cual, se logra a partir de la modificación geométrica de la división de los puertos. esto se comprobó con los resultados de la buza modificada.

\section{BIBLIOGRAFÍA}

[1] HONEYANDS, T., HERBERTSON, J. "Flow Dynamics in Thin Slab Caster Moulds", Steel Research, v.66, n.287, 1995.

[2] GUPTA, D., LAHIRI, A. K. "Water-Modelling Study of the Surface Disturbances in Continuous Slab Caster", Metallurgical and Materials Transactions B, v. 25, n.2, pp. 227-233, 1994.

[3] GUPTA, D., LAHIRI, K. "A Water Model Study of the Flow Asymmetry Inside a Continuous Slab Casting Mold", Metallurgical and Materials Transactions B, v. 27, n.5, pp.757-764, 1996.

[4] ASM HANDBOOK. Chapter 15 in Modeling for Casting and Solidification Processing, New York, NY, pp. 499-540, 2001.

[5] TRIPPELSDORF, H., MARRACINI, R., KOLLBERG, S. "Advances in Strip Surface Quality from Thin Slab Casters", International Scientific Colloquium, Modelling for Electromagnetic Processing, Hannover, March 24-26, pp.189-196, 2003.

[6] MORALES, R. D., PALAFOX RAMOS, J., GARCIA, L., et al., "A DPIV Study of Liquid Steel Flow in a Wide Thin Slab Caster Using Four Ports Submerged Entry Nozzles", ISIJ International, v. 44, n. 8, pp. 1384-1392, 2004.

[7] SHEN, B., SHEN, H., LIU, B. "Inestability of Fluid Flow and Level Fluctuation in Contonuous Thin Slab Casting Mould", ISIJ International, v. 47, n. 3, pp. 427-432, 2007.

[8] ALONSO, E.T., MORALES, R. D., DEMEDICES, L.G., et al., "Flow Dynamics in Thin Slab Molds Driven by Sustainable Oscillating Jets from the Feeding SEN", ISIJ Int., v.47 , 679, 2007.

[9] LIU, H., YANG, C., ZHANG, H., et al., "Numerical Simulation of Fluid Flow and Thermal Characteristics of Thin Slab in the Funnel-Type Molds of Two Casters", ISIJ International, v. 51, n. 3, pp. 392-401, 2011.

[10] SHEN, B., SHEN, H., LIU, B. "Water modelling of level fluctuation in thin slab continuous casting mould", Ironmaking and Steelmaking, v. 36, n. 1, 2009.

[11] TORRES-ALONSO, E., MORALES, R. D., GARCÍA HERNÁNDEZ, S., et al., "Cyclic Turbulent Instabilities in a Thin Slab Mold. Part I: Physical Model”, Metallurgical and Materials Transactions B, v. 41B, pp.583-597, 2010.

[12] LI, B., TSUKIHASHI, F. "Effects of Electromagnetic Brake on Vortex Flows in Thin Slab Continuous Casting Mold", ISIJ International, v. 46, n. 12, pp. 1833-1838, 2005.

[13] TORRES-ALONSO, E., MORALES, R. D., GARCÍA HERNÁNDEZ, S. "Cyclic Turbulent Instabilities in a Thin Slab Mold. Part II: Mathematical Model", Mathematical Model" Metallurgical and Materials 
Transactions B, v. 41, pp.675-690, 2010.

[14] ARCOS-GUTIERREZ, H., BARRERA-CARDIEL, G., BARRETO, J.J., et al., "Quantitative Determination of Free Lime Amount in Steelmaking Slag by X-ray Diffraction", ISIJ International, v.54, n.6, pp. 1334-1337, 2014.

[15] POPE, S. B. Turbulent Flows, Cambridge University Press, Cambridge, London, New York, 2000.

[16] ANDERSSON, B., ANDERSSON, R., HAKANSSON, L., et al., Computational Fluid Dynamics for Engineers, Cambridge University Press, Cambridge, London, New York, 2012.

[17] WILCOX, D. C. Turbulence Modeling for CFD, DCW Industries Inc.,La Canada, California, 2006.

[18] Schlichting, H., Gersten, K. Boundary Layer Theory, Springer-Verlag Berlin Heidelberg, 2000.

[19] FLUENT 6.2. User's Guide. Fluent Inc., Lebanon, New Hampshire, USA, Chapter 12 and 23, 2005.

\section{Nomenclatura.}

$\mathrm{g}=$ Aceleración gravitacional $\left(\mathrm{m} / \mathrm{s}^{2}\right)$

$\mathrm{k}=$ Energía cinética turbulenta $\left(\mathrm{m}^{2} / \mathrm{s}^{2}\right)$

$\mathrm{P}=$ Presión $(\mathrm{Pa})$

$\mathrm{u}=\operatorname{Velocidad}(\mathrm{m} / \mathrm{s})$

$\mathrm{U}^{\prime}=$ Velocidad fluctuante $(\mathrm{m} / \mathrm{s})$

$\operatorname{Re}_{\mathrm{T}}=$ Reynolds turbulento (-)

Símbolos Griegos

$\rho=\operatorname{Densidad}\left(\mathrm{kg} / \mathrm{m}^{3}\right)$

$\mu=$ Viscosidad dinámica $(\mathrm{Pa} \mathrm{s})$

$\mu_{\text {eff }}=$ Viscosidad efectiva (Pa $\left.s\right)$

$\mu_{\mathrm{t}}=$ Viscosidad turbulenta $(\mathrm{Pa} \mathrm{s})$

$\nabla \cdot=$ Divergencia $(-)$

$\nabla=$ Gradiente (-)

$\varepsilon=$ Relación de disipación de energía cinética turbulenta $\left(\mathrm{m}^{2} / \mathrm{s}^{3}\right)$

$\rho\left\langle U_{\mathrm{i}}^{\prime} U_{\mathrm{j}}^{\prime}\right\rangle=$ Esfuerzos de Reynolds (-) 